\title{
La paz en el ethos de la universidad
}

Manuel José Jiménez Rodríguez ${ }^{1}$.

Recibido:

Febrero 20 de 2012

Aceptado:

Mayo 25 de 2012
${ }^{1}$ Doctor en teología y pedagogía religiosa de la Pontificia Universidad Salesiana de Roma. Posdoctorado con la Red CLACSO y el CINDE

de Manizales. Investigador y miembro del equipo pedagógico nacional de la Corporación

Observatorio para la paz de la ciudad de Bogotá, Colombia.

El presente estudio hace parte de una investigación realizada dentro del postdoctorado EN Investigación en Ciencias Sociales, Niñez y Juventud, ofrecido por Centro de Estudios Avanzados en Niñez y Juventud de la alianza CINDE - Universidad de Manizales - Colombia y la Pontificia Universidade Católica de São Paulo - Brasil, con el aval del Consejo Latinoamericano de Ciencias Sociales - CLACSO.

\section{Resumen}

La UNESCO, la sociedad en general y el mismo sistema educativo en su conjunto y niveles, reconocen en la educación un gran aporte a la cultura de paz. La universidad, dentro del sistema de educación superior, no es ajena a estos llamados de educar para la paz. Tanto los encuentros mundiales, como estudios recientes de la situación actual y futura de la universidad, son ejemplos de dicha preocupación.

En este estudio se quiere mostrar las distintas maneras como la paz puede hacerse presente en la universidad, buscando señalar algo que alguien dijo acerca de la universidad católica, pero que cabe, y debe ser algo propio, de toda universidad por el hecho mismo de ser universidad. Lo dicho fue: "La paz se encuentra en el corazón de la universidad"

Palabras clave: Universidad, paz, educación para la paz, cultura académica, cultura de paz, reforma de mentalidades.

\section{Abstract}

UNESCO, society in general and the same educational system in its entirety and levels, recognize a great contribution to the culture of peace in education. The University, within the system of higher education, is no stranger to these socalled of educating for peace. Both global events, and recent studies of the current situation and future of the University, are examples of such concern.

This study wants to show the different ways as peace can become present in the University, looking for point out something that someone said about the Catholic University, but that should be, and must be something own of all University by the very fact of being University. What was said was: "peace is located in the heart of the University"

Keywords: University, peace, education for peace, culture, academic, culture of peace, reform of mentalities 


\section{Introducción}

L a UNESCO, la sociedad en general y el mismo sistema educativo en su conjunto y niveles, reconocen en la educación un gran aporte a la cultura de paz. Basta a este respecto ver varios pronunciamientos de la UNESCO, desde sus propios preámbulos, para darse cuenta que la relación estrecha entre educación y paz es asunto de primer orden de cara a la transformación de la sociedad y de los mismos seres humanos.

La universidad, dentro del sistema de educación superior, no es ajena a estos llamados de educar para la paz. Tanto los encuentros mundiales, como estudios recientes de la situación actual y futura de la universidad, son ejemplos de dicha preocupación. Con todo y la importancia señalada, no faltan estudios que hacen ver otra cara de la moneda. Distintas instituciones, ámbitos escolares, medios de comunicación y familia, socializan hoy a los seres humanos en una mentalidad militarista que en la misma paz. Mentalidad desde la cual se acepta la violencia como algo natural y necesario, y se generan cantidad de miradas sobre la vida y el otro desde lógicas violentas y no de paz.

El reto educativo es doble. Por un lado educar en paz y sus valores, y por otro transformar dicha mentalidad militarista tan común y hasta más arraigada que la misma paz.

Para el caso de la universidad, la relación con la paz suele ser abordada desde distintas perspectivas, que son complementarias, pero que en muchos casos y en la práctica se le da más importancia a una sobre otras, como suele ser el darle más realce a los estudios e investigaciones que sobre la paz, la violencia y la guerra se hacen en la universidad y algunos centros de estudios, que la misma cultura o ambiente de paz que es propio de la universidad y de su ethos académico.

En este estudio se busca señalar algo que alguien dijo acerca de la universidad católica, pero que cabe, y debe ser algo propio, de toda universidad por el hecho mismo de ser universidad. Lo dicho fue: "La paz se encuentra en el corazón de la universidad". ${ }^{2}$

Propósito de este breve estudio es llegar a descubrir la paz en el corazón de la universidad. Paz que no puede ser reducida a una de sus muchas concepciones, sino que las abarca cada una de ellas, especialmente las relacionadas con la paz negativa (la contraria a la guerra y a la violencia directa), la paz positiva (la opuesta a la violencia estructural), la paz como cultura(la que tiene que ver con las representaciones y cosmovisiones) y la cultura de paz en los términos señalados por la UNESCO en sus distintos estudios. Es decir, la que guarda relación estrecha con los derechos humanos, el desarrollo, la democracia, la ciudadanía, el problema ecológico y la cuestión de género.

La paz en la universidad: distintas relaciones $y$ posibilidades.

En un breve capítulo de un libro dedicado al estudio de la educación para la paz, el estudioso español Alfonso Fernández Herrería, analiza dos sentidos que puede tomar la educación para la paz en la universidad. ${ }^{3}$ Uno, entendiendo la educación para la paz como materia o asignatura, relacionada con materias o con facultades cercanas a las temáticas de la paz. Dos, entendiéndola como educación en paz, es decir, como elemento fundamental y común a todo el hecho educativo en su conjunto y, por lo tanto, a toda asignatura.

La segunda forma, la llama este autor educación para la paz como forma o

${ }^{2}$ Guy-Réal THIVIERGE, Au coer de l'universitácatholique: L'education a la paix, en "Seminarium" 3/4 (2001) 797 - 824.

${ }^{3}$ Alfonso FERNÁNDEZ HERRERÍA, La educación para la paz en la universidad, en Javier Rodríguez Alcázar (editor), Cultivar la paz. Perspectivas desde la universidad de Granada, Universidad de Granada, Granada 2000. 
estructura. En este caso, la paz y sus valores se hacen presentes en todo el sistema educativo. La educación en los valores de la paz debe desarrollarse en la forma de hacer educación. En otras palabras, en educación en paz debe haber coherencia entre los medios y los fines. De modo tal que no puede decirse que se educa en, desde y para la paz con medios que sean totalmente contrarios a la paz y sus valores.

Otras dos formas de entender la paz en la universidad la ofrece el filosofo AngeloPapacchini. ${ }^{4}$ Uno, es la que puede llamarse estudios de la paz desde y en la universidad. ${ }^{5}$
Que para el caso colombiano es un asunto emblemático y de primer orden como lo muestran algunos congresos sobre universidad y paz. ${ }^{6}$

Dos, es la paz presente en el ethos académico y sus valores. Para este autor la universidad y su ethos o cultura académica que la caracteriza es un ejemplo para la sociedad en cuanto al manejo no violento de los conflictos. De modo tal que dicho ethos es más cercano a la paz, sus valores y sus lógicas, que a los de la violencia y la guerra.

Y una última forma de ver la relación pazuniversidad guarda relación con la pertinencia y la responsabilidad social de la universidad, ${ }^{7}$

\footnotetext{
${ }^{4}$ Ángelo PAPACCHINI, Universidad, guerra y paz, en Carmen Lucia DIAZ - Claudia MOSQUERA - Fabio FAJARDO (Compiladores), La universidad piensa la paz: obstáculos y posibilidades, Universidad Nacional de Colombia, Bogotá 2002, 15-49.
}

${ }^{5}$ Puede decirse que en Colombia se han dado diversas modalidades de asumir la universidad su compromiso frente al conflicto armado interno y la paz. Modalidades que también pueden ser reconocidas por universidades en otros contextos distintos al colombiano, pero también con situación de conflicto interno, de acuerdo con una investigación llevada a cabo por la universidad Jorge Tadeo Lozano de Bogotá Colombia.Según dicha investigación, las modalidades son las siguientes. Primero, desde un sentido de Responsabilidad Social tradicional, labores de investigación, docencia y extensión orientadas a la construcción de paz. Se encuentran acciones de carácter preventivo dirigidas a prevenir situaciones de conflicto y violencia a través de difusión de información en seminarios, foros y cátedras en cultura de paz, que se conciben también como espacios de discusión y reflexión sobre el posconflicto. También se encuentran las actividades de carácter curricular que tienen por objeto formar no sólo a la población estudiantil, sino a diversos sectores de la sociedad en temas concernientes a la situación de conflicto como el marco jurídico y político de los acuerdos de paz, los derechos humanos, y las experiencias internacionales significativas en construcción de paz, entre otros. Para el caso particular de las acciones preventivas se puede señalar también la función que cumplen los centros de investigación, cuya función es la de pensar y analizar desde una perspectiva académica y del conocimiento las raíces estructurales del conflicto y las posibles salidas al mismo. Segundo, desde una concepción de Responsabilidad social orientada a la resolución pacífica del conflicto armado, acciones de naturaleza paliativa, orientadas a manejar la situación de conflicto, mediante la búsqueda de salidas o caminos posibles al mismo. Dentro de este grupo de acciones puede ser clasificados los esfuerzos de las universidades encaminados a "acercar" a las partes en conflicto a través de una mediación , facilitación, buenos oficios y el arbitramiento, o los esfuerzos de la institución cuando ésta se declara "agente de paz" y al campus universitario un espacio neutral en el conflicto. Tercero, desde una comprensión de responsabilidad social orientada a promover las condiciones pacíficas de existencia de la población durante el conflicto o a garantizar la sostenibilidad de las condiciones de existencia de la población en el posconflicto. Es posible encontrar acciones de tipo restaurativo: iniciativas que se crean con el propósito de contribuir a "sanar" las heridas o secuelas del conflicto y en términos más amplios a reconstruir el tejido social a través de acciones que busquen la reconciliación no sólo entre los actores en conflicto sino entre víctimas y victimarios. Dentro de estas iniciativas se encuentran también los proyectos enfocados a posibilitar una adecuada reinserción de los ex combatientes a la vida civil. (CfMariana DELGADO BARÓN - Yaneth Angélica VARGAS PEDRA ZA - Ivonne RAMOS HENDEZ, Los retos de la responsabilidad social universitaria. Construyendo paz desde la universidad, en Revista educación superior y sociedad", Número 2, septiembre de 2008, 64 -91)

${ }^{6} \mathrm{~A}$ este respecto puede verse ASOCIACIÓN COLOMBIANA DE UNIVERSIDADES (ASCUN), La universidad y la paz. Consejo nacional de rectores 30 de septiembre - 1 de octubre, 1999, ASCUN, Santafé de Bogotá 1999; ASOCIACIÓN COLOMBIANA DE UNIVERSIDADES POR LA PAZ (ASCUN) - RED DE UNIVERSIDADES POR LA PAZ (REDEUNIPAZ), Primer congreso universitario por la paz, tomo I y II, Santa fe de Bogotá D.C. 12, 13 y b14 de mayo de 1999, ASCUN, Santa fe de Bogotá D.C., 2000; INSTITUTO COLOMBIANO PARA EL FOMENTO DE LA EDUCACIÓN SUPERIOR (ICFES), Memorias del Congreso Nacional de Educación Superior. Eje temático: proyecto nación. Reuniones realizadas el 1 de diciembre de 1999, ICFES, Bogotá D.C 2000; Lucia DIAZ CARMEN - Claudia MOSQUERA - Fabio FAJARDO (Compiladores), La universidad piensa la paz: obstáculos y posibilidades, Universidad Nacional de Colombia, Bogotá D.C. 2002.

${ }^{7}$ La Responsabilidad Social es un nuevo modo de gestión de las organizaciones que se basa en estándares éticos internacionalmente reconocidos para la promoción de "buenas prácticas", tanto en la administración interna de la organización (gestión laboral y medioambiental) como en su vínculo con la sociedad (gestión de la relación social). Es la disciplina ética que nos recuerda nuestros deberes no sólo para con nuestras acciones, sino para con el campo en el cual se desarrollan, ensanchando así nuestras obligaciones morales hacia el cuidado de todo el sistema: la sostenibilidad de toda la casa Tierra, con todos sus habitantes y todas sus sociedades humanas. Es ética, porque la ética que le corresponde a la Responsabilidad Social no es una ética personal que limita la responsabilidad a la pequeña esfera de los actos voluntarios del individuo. Es una ética social, coordinada y consensuada entre actores sociales para la solución de problemas sociales.Con todo ello, la meta de la responsabilidad social es la transformación de nuestro modo de existir en el planeta. Por ello concierne a todos en todo el mundo, porque promueve una gestión justa y sostenible de nuestra vida en el planeta.François Vallaeys define la responsabilidad social universitaria de la siguiente manera: Es una política de mejora continua de la Universidad hacia el cumplimiento efectivo de su misión social mediante cuatro procesos: Gestión ética y ambiental de la institución; Formación de ciudadanos responsables y solidarios; Producción y Difusión de conocimientos socialmente pertinentes; Participación social en promoción de un Desarrollo más humano y sostenible. La Responsabilidad Social Universitaria procura alinear los cuatro procesos universitarios básicos de Gestión, Formación, Investigación y Extensión con las demandas científicas, profesionales y educativas que buscan un desarrollo local y global más justo y sostenible. François VALLAEYS, Responsabilidad social empresarial, enhttp://www.iesalc.unesco.org.ve /;François VALLAEYS, Responsabilidad social universitaria: una nueva filosofía de gestión ética e inteligente para las universidades, en "Revista educación superior y sociedad", Número 2, septiembre de 2008, 191-220. 
en las actuales sociedades globalizadas, del conocimiento y de amplia injusticia social y violencia.

Para algunos estudiosos, como Cristina De La Cruz Ayuso y Perú Sasia Santos, lo que no apunte a una respuesta concreta sobre la responsabilidad de la Universidad en un contexto de injusticia, desigualdad, inequidad, conflicto y exclusión, es un falso debate y una débil aproximación a la responsabilidad de las universidades con la sociedad. ${ }^{8}$ De modo tal, que la universidad no sólo es responsable frente a sí misma, lo es también frente a la sociedad. Es responsable no sólo de quienes acuden a sus aulas o se forman en ella, o trabajan en ella como docentes o como administrativos. No se trata solamente de que la Universidad deje de ser ella misma también un agente de exclusión, sino que también se convierta en un agente de transformación de nuestras sociedades, una organización efectivamente comprometida en la tarea de hacer retroceder las fronteras de la injusticia. ${ }^{9}$

En los párrafos que siguen no se abordan estos múltiples sentidos y presencias de la paz en la universidad, sino sólo el que guarda relación con su cultura académica o ethos cultural. Aunque no se toque, no se puede olvidar que lo dicho aquí debe leerse al interior del actual contexto de la universidad y su necesaria modernización hoy, de modo que sea más pertinente en y para las actuales sociedades globalizadas y del conocimiento, con el riesgo de reducir la universidad, su pertinencia y responsabilidad social a las lógicas del mercado. procesos de transformaciones sociales, lo hace desde aquello que le es propio, es decir, como universidad, no como partido político o como cualquier otra institución social, en donde el factor primordial es el conocimiento. Pero no cualquier tipo de conocimiento, sino un conocimiento crítico. Es colocar su acerbo de conocimientos al servicio de los trabajos e investigaciones que requiere el país para resolver sus tan graves problemas estructurales y coyunturales.

La universidad actúa así sobre la sociedad desde su propio "Ethos cultural", que es lo académico, atravesado a su vez por las misiones tradicionales de la universidad, a saber: la enseñanza (docencia, formación), la investigación y la extensión. También implica participar y educar desde los valores propios de su "ethos cultural y académico", como son el pluralismo de ideas, la controversia intelectual, la discusión pacífica, la diversidad y la divergencia, la participación democrática. Valores todos lejanos a las lógicas de violencia y más bien acordes a la paz y sus lógicas. El Ethos universitario es así radicalmente opuesto al sectarismo, a la exclusión, a la exacerbación de los mecanismos de fuerza para obtener el poder. Sus valores son los de la formación por el razonamiento y la argumentación, la diversidad de opiniones, la solidaridad y la aceptación de la diferencia como riqueza. A este respecto afirma Angelo Papacchini: "La universidad tiene que enfrentar los problemas concretos de su tiempo y del contexto específico en el que se inscribe, pero con sus herramientas más peculiares: el conocimiento, la investigación científica, la crítica y un ethos sustentado en el dialogo y en el poder de la palabra, la autonomía, el juicio imparcial y el compromiso solidario con la dignidad de todo ser humano". ${ }^{10}$

La participación de la universidad en estos

${ }^{8}$ Cristina DE LA CRU Z AYUSO - Perú SASIA SANTOS, La responsabilidad de la universidad en el proyecto de construcción de una sociedad, en "Revista educación superior y sociedad", Número 2, septiembre de 2008, 17- 52.

${ }^{9}$ Para estos estudiosos, es responsabilidad de la universidad la promoción de la justicia social, es decir, la transformación de las estructuras de injusticia y desigualdad de nuestras sociedades a partir de los ámbitos de actividad que le son propios. Lo cual implica poner la docencia, la investigación y la proyección social al servicio de la justicia social. De ahí que se pregunten acerca del ser y del quehacer de la universidad hoy: "ibasta con que la universidad responda a una sociedad - no lo olvidemos que sigue excluyendo? ¿Quién decide, y en base a qué criterios, que es aquello a lo que una universidad debe responder? ¿Cabe acaso pensar que la universidad es responsable de orientar esa respuesta no solamente para que intervenga en la sociedad, sino para que la transforme?"

${ }^{10}$ Ángelo PAPACCHINI, Universidad, guerra y paz 17. 
La tarea de la universidad de "pensar la paz" desde su propio "ethos", exige también una "reconversión" o transformación de la universidad en su cultura organizacional académica a la paz y sus lógicas. Es decir, que la responsabilidad de la universidad frente a la transformación del país y la paz pide de ella su transformación institucional. La paz pide de la universidad un repensamiento de la sociedad desde las lógicas de la paz y exige, al mismo tiempo, un repensamiento de la universidad en sí misma, en sus procesos institucionales, organizacionales y académicos.

Se trata de que la paz y sus valores inspiren los criterios de organización de la universidad y su "ethos"académico. ${ }^{11}$ De este modo la universidad, nuevamente lo señala Angelo Papacchini, ofrece a toda la sociedad un ejemplo de comunidad abierta y dinámica, en la que no se nieguen o repriman los conflictos, sino que se canalicen de acuerdo con principios mínimos de convivencia.

Todo lo dicho permite concluir que la paz pertenece a la entraña misma de la universidad. La universidad por ser universidad es el lugar por excelencia del pluralismo, del dialogo, de la convivencia, de la mirada crítica y analítica sobre la realidad. Tiene como misión primordial la formación integral del ser humano, dentro de la cual se comprende su formación profesional y académica. La paz y su educación, afirma Guy-RéalThivierge, están en el corazón mismo de toda universidad. A través de la enseñanza, del debate, la investigación, el diálogo, la universidad educa a la paz. Ella educa a la paz en el ejercicio de todas sus funciones: culturales, sociales, políticas $^{12}$. Ella es por excelencia ambiente donde se educa a la paz, o en palabras de Angelo Papacchini "espacio y taller de paz".

\section{La cultura de paz y los valores de la cultura académica}

Desde la racionalidad comunicativa la universidad es vista no sólo como institución sino también como una comunidad sui generis, en la que sus miembros han de buscar relacionarse entre sí, con el saber y con su sociedad. Se trata de una racionalidad que permite comprender la educación no sólo como una acción orientada al éxito, sino ante todo al entendimiento. Pero no cualquier tipo de entendimiento, sino al entendimiento sobre bases racionales, o sea, mediante un trabajo reflexivo en común, en donde la ambigüedad y el conflicto son dimensiones centrales de la vida académica y cultura académica.

La cultura académica universitaria posee una serie de valores propios, que son sus valores académicos. Que si bien son comunes a todos los subgrupos sociales, según el rector de la universidad de Sao Paulo Jacques Marcovich, "en la institución académica constituyen un bagaje esencial para el buen cumplimiento de su misión”.Y estos son, en su pensamiento, los siguientes: pluralismo, universalismo, solidaridad ética y excelencia. ${ }^{13}$

El pluralismo es el valor específico de la institución académica. En la universidad, la libre circulación de las ideas es su propia razón de ser. Han de transitar en ella las más diversas y variadas corrientes de opinión. La búsqueda del saber exige esa continua apreciación desde

\footnotetext{
${ }^{11}$ XesusJares es quizás uno de los autores contemporáneos que más ha estudiado todo lo relacionado con la educación para la paz. Uno de sus escritos tiene como centro de análisis la relación entre educación para la paz y organización escolar. Hacemos referencia a esto pues nos permite comprender la importancia de superar una concepción muy reducida de educación para la paz vista solo como información, y entenderla de un modo más integro e integral, que es la propuesta que esta detrás de toda la concepción de AngeloPapacchini y de muchos otros autores contemporáneos. Una pregunta atraviesa todo su estudio: $i$ Los objetivos, organización, relaciones y actitudes presentes en la escuela, refuerzan o socavan los objetivos y enfoques propios de la educación para la paz?. De ahí la insistencia que haga acerca de la inevitable relación entre educación para la paz y organización de los centros escolares, pues no puede haber incoherencia entre los medios y los fines. Esto lo lleva a afirmar que la escuela (la universidad) no sólo educa para la paz, sino que también educa sobre la paz y en paz. Se trata así de superar los valores de competitividad y de lógica de guerra que rigen el sistema educativo actual. (Cf Xesus JARES, Educación para la paz y organización escolar, en Alfonso FERNÁNDEZ HERRERÍA (editor), Educación para la paz: nuevas propuestas, Universidad de Granada, Granada 1994, 285 - 316. )

${ }^{12}$ Guy-Réal THIVIERGE, Au coer de l'universitácatholique: Leducation a la paix, en "Seminarium” $3 / 4$ (2001) 797 - 824.

${ }^{13}$ Jacques MARCOVICH, La universidad (im)posible, Cambridge UniversityPress - Organización de Estados Americanos (OEI), Madrid $2002,29$.
} 
todos los ángulos, en todas las cuestiones. "El pluralismo, abrigando tendencias dispares, no presupone en absoluto un pozo de contradicciones. La pasión intelectual es el hilo invisible que los une a todos. El respeto por las convicciones ajenas prevalece en cualquier circunstancia, incluso en el más agudo conflicto de ideas". ${ }^{14}$

Profundizando en el ethos académico, el filosofo colombiano Guillermo Hoyos, también reconoce en el pluralismo un valor clave y fundamental en la universidad. Y entiende por éste una universidad que no se resiste a las confrontaciones radicales de formas de vida y de pensamiento casi incompatibles. Ella es un espacio apto para la divergencia de pensamiento, de conductas, de estilo de vida y de estilos científicos. La universidad es así lugar del desacuerdo obligado y su responsabilidad central es iniciar a los estudiantes al conflicto. A la vez debe animar la controversia con otros pareces rivales: así se podrá ver lo que está equivocado en ellos desde nuestro punto de vista, como lo que está equivocado en el nuestro desde las objeciones de los oponentes. Esto significa, subraya citando a MacIntyre, que es tarea prioritaria de la universidad asegurar que las voces rivales no sean suprimidas, que ella no sea una arena neutral de objetividad, sino más bien arena de conflicto en la que se otorgara reconocimiento al tipo más fundamental de desacuerdo moral y filosófico. ${ }^{15}$

En la regulación pacífica de sus conflictos académicos, la universidad ha de presentarse ante la sociedad como ejemplo de respeto y tolerancia, tal como lo afirma el filósofo Angelo Papachini al hablar en un congreso sobre la universidad y la paz: "Creo que la universidad debería ofrecer un ejemplo de comunidad abierta y dinámica, en la que no se nieguen o repriman los conflictos, sino que se canalicen de acuerdo con principios mínimos de convivencia. En últimas, la universidad debería desempeñar una función crítica de lo que es, pero también empezar a materializar algo lo que debe ser; y mostrar que la convivencia pacífica sustentada en el respeto es algo más que una utopía lejana y puede encontrar lugar en nuestra realidad. El espacio universitario debería así funcionar como un auténtico taller de relaciones no libres de conflictos, pero orientadas por valores como la dignidad, el respeto y la solidaridad, que deberían inspirar por igual las prácticas pedagógicas, el trabajo investigativo y la convivencia entre los diferentes estamentos de la universidad (...). La universidad debería ofrecerle a la sociedad en general un ejemplo de controversias y disputas limpias entre sujetos y grupos que difieren en cuanto a principios, cosmovisiones y paradigmas científicos, y sin embargo comparten normas mínimas en cuanto al juego limpio y a la igual oportunidad para todos de argumentar y luchar para defender un enfoque epistemológico, ético o científico consolidado a través de años de investigación y docencia; debería ofrecer el ejemplo de sujetos dispuestos no solamente a tolerar, sino a interesarse activamente por posturas diferentes". ${ }^{16}$

La universidad es así lugar en donde se ejerce la auténtica democracia y educadora de la democracia. Y lo es porque la racionalidad comunicativa es el factor determinante de todo proceso educativo, especialmente el universitario. Por su apelación constante al diálogo y a la discusión racional y porque en ellas las diferencias no se resuelven por la vía de la violencia. Esta no es una tarea que le sea impuesta o que ella misma se exija a cumplir: le basta con hacer bien lo que tiene que hacer para que, sin proponérselo expresamente, contribuya a la democracia.

\footnotetext{
${ }^{14}$ Jacques MARCOVICH, La universidad (im)posible 29.

${ }^{15}$ Guillermo HOYOS, El ethos de la universidad, en "Revista UIS-Humanidades 1 (1998) 13 - 23.

${ }^{16}$ Ángelo PAPACCHINI, Universidad, guerra y paz, en Carmen Lucia DIAZ - Claudia MOSQUERA - Fabio FAJARDO (Compiladores), La universidad piensa la paz: obstáculos y posibilidades, Universidad Nacional de Colombia, Bogotá 2002, 41.
} 
La universidad es un lugar democrático por excelencia, en donde en su práctica pedagógica en los campos de la docencia, la investigación y la extensión, así como en su forma de organización y administración, han de primar los valores del respeto a las diferencias, el diálogo, la tolerancia, el compromiso social, las soluciones razonadas, la participación, entre muchos otros. Todos ellos, en contra de la violencia, la imposición y la mentira.

Por ser plural, en la universidad ha de regir también el valor de la solidaridad social. La solidaridad característica de la cultura académica es la propia de las sociedades modernas, es decir, aquella que desarrolla y tolera cantidad y variedad de interpretaciones heterogéneas. En este sentido la universidad ha de multiplicar las lecturas y afinarlas mediante la crítica y la contrastación. La universidad apoya los procesos de modernización de las sociedades, responde a los valores fundamentales de la globalización (pluralismo y diversidad) y apoya el cambio en los principios de solidaridad, que deja de ser "mecánica" (basada en credos e interpretaciones compartidas) y pasa a ser "orgánica" acompañada por la proliferación de credos, la proliferación de posibilidades de lectura.

Como complemento y cierre a esta amplia relación entre los valores de la cultura de paz y los de la cultura académica aplicados a la universidad, vale la pena traer a colación a uno de los estudiosos de la educación en América Latina, el pedagogo brasileño Paulo freire. Quien en un pequeño estudio, si bien resalta los valores que él considera que son característicos de la universidad católica posmodernamente progresista, afirma que dichos valores son propios de toda universidad, no sólo de la católica en particular. ${ }^{17}$

En primer lugar, señala la tolerancia. Y la define como la virtud cuya práctica nos enseña a convivir con lo diferente, sin que eso signifique que los diferentes desistan de continuar defendiendo sus posiciones. La tolerancia significa, palabras suyas, únicamente que los diferentes tienen derecho a seguir siendo diferentes y de aprender de sus diferencias. La tolerancia no pretende negar ni tampoco esconder los posibles conflictos entre los diferentes, ni por otro lado desconocer que hay diferentes que son más que diferentes porque son antagónicos entre sí. Lo que la tolerancia pretende es la convivencia posible, respetadas las diferencias de los que conviven.

De modo que, una universidad, subraya Freire, es más democrática cuanto más tolerante es, cuanto más se abre a la comprensión de los diferentes, cuanto más se torna en objeto de la comprensión de los demás. Pues es la tolerancia en su forma descrita, la que debe informar las distintas tareas de la universidad, la docencia, la investigación, la extensión; las relaciones entre las facultades, las relaciones entre los distintos departamentos y programas, así como entre las personas.

La segunda virtud o valor es la humildad. La tolerancia, piensa Freire, es imposible sin la humildad. La humildad es todo lo contrario a sobreestimarme o a subestimar al otro. Por eso, es contraria a la arrogancia, que acompaña más bien la intolerancia.

La tercera, consiste no sólo en manifestar sino vivir la permanente búsqueda de la pasión por la curiosidad. No se trata, de la curiosidad "desarmada", sino de la curiosidad metódica, exigente, epistemológica, que va muy unida al proceso de des-ocultamiento de la verdad. Tareas que además, no han de ser intolerantes. Como lo afirma Freire: "Me siento satisfecho en mi misión de educador cuando, peleando para convencer a los educandos de lo acertado de mi des-ocultación, me vuelvo yo mismo transparente al revelar, primero, mi respeto por el posible rechazo de los educandos a mi discurso, y segundo mi respeto por su anti-

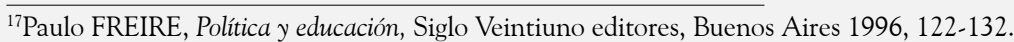


verdad, con la cual me niego a convivir: $\mathrm{Me}$ siento satisfecho en mi misión de educador, por último, cuando revelo mi tolerancia frente a los que son diferentes de mí. Por el contrario, desmerezco de mi misión de educador y ante mí mismo si, en nombre del respeto a los educandos, callo mis posiciones políticas y mis sueños, o si pretendo, en nombre de mi autoridad de educador, imponerles mis criterios de verdad". ${ }^{18}$

\section{"La paz en el corazón de la universidad"}

Este apartado de algún modo puede ser considerado un apartado "síntesis", ya que permite ahondar en una afirmación que hasta ahora se ha hecho expresa de un modo muy general: "la paz se encuentra en el corazón de la universidad". Y es "síntesis" por varias razones. Porque va a permitir poner en dialogo algunos aspectos que sobre la universidad se han dicho en apartados anteriores, a saber: el aporte de la universidad a la construcción de una globalización de rostro humano y solidario, la pertinencia social de la universidad y su función de ser conciencia crítica de la sociedad, el papel de la universidad en Colombia frente al conflicto interno armado y la paz, la "racionalidad" (razón, saber y conocimiento) como factor estructurante del "secreto" de la universidad, y los valores del "ethos académico" de la universidad como valores propios de la cultura de paz.

Todo lo anterior exige superar la lógica de mercado y su racionalidad tecnocrática en el modo de concebir la misión, uso y funciones de la universidad. Desplazando de este modo su peso y eficiencia social. La universidad pierde así y se aleja de su misión de ser portadora de una función crítica del orden establecido, función que desde la teoría es señalada y defendida por los distintos foros de educación suprior y universidad, como los de París y la Habana. Lo que conllevaría a que se pierda la posibilidad de que en la universidad tengan acceso los otros "muchos modos de conocer" y así deje de lado su función de crítica y construcción de un futuro alternativo y diferente para la humanidad.

Para evitar el riesgo de pensar que todas las tareas relacionadas con la paz y la transformación de las sociedades sean consideradas como algo accesorio, marginal y añadido, se hace necesario pensarlas desde el "secreto" de la universidad", su deber primordial, ligado al conocimiento y la racionalidad comunicativa. De ahí, recordando lo dicho en su momento, que el aporte de la universidad a la sociedad, al estado, a la cultura, a la transformación de la globalización, al desarrollo justo, desde su cultura académica es la racionalidad, tal como lo subraya el pensador colombiano Antanas Mockus.

En donde racional, según el parecer de Mockus, quiere decir basado precisamente en la acción comunicativa discursiva, en la tradición escrita y en la elaboración de lo que se ha aprendido en los intentos previos de reorientar o reorganizar acciones semejantes. Donde racional significa huir de los dogmatismos, de las posiciones radicalmente subjetivas y autoritarias sin más fundamento que el "porque así lo pienso" yo, y asumir opiniones personales razonadas y razonables frente a los problemas de la sociedad o comunidad de la que hace parte, gracias a la cultura y al conocimiento adquirido en la universidad. ${ }^{19}$

Pensada así, la universidad es un tipo particular de microsociedad en la que es posible, mediante el recurso a la acción comunicativa discursiva, evaluar racionalmente argumentos y cuestionar toda pretensión a la verdad, sin que por ello se rompa la comunicación ni se termine la cooperación entre sus miembros. De este, afirma Mockus, alguien realmente formado

\footnotetext{
${ }^{18}$ Ibid 131 .

${ }^{19}$ Antanas MOCKUS SIVICKAS, Fundamentos teóricos para una reforma de launiversidad, en ESCUELA SUPERIOR DE ADMINISTRACIÓN PUBLICA, Reflexionessobre el sentido de la universidad, ESAP, Bogotá 1992, 97 - 125.
} 
en la cultura académica es alguien que se convierte en una especie de "racionalizador", en alguien que no se conforma, es alguien que ha sido sacado de la evidencia de lo inmediato, de lo acostumbrado; que se ha abierto a la posibilidad de preguntar: ¿por qué siempre ha sido así? ¿Cómo podría ser de otro modo? Es alguien que hace posible y obligatoria la exploración de alternativas.

Claro que se habla una racionalidad amplia y abierta, más allá de la racionalidad positivista que reduce la ciencia al método empírico positivo, que solo llega a aceptar como existente lo que es comprobable por la ciencia positiva. Y la universidad construye la paz desde esa razón.

Para comprender lo dicho se hace necesario tener claro los cambios producidos tantos en los conceptos de paz y de razón a lo largo de la historia, y a la par de estos la comprensión de la misión de la universidad. ${ }^{20}$ Por ejemplo, en la Edad media, la idea de paz se encuentra conectada con la idea de concordia. La paz se concibe como armonía, particularmente entre las personas, pero también es armonía del alma, armonía interior. Desde esta tradición se pensaba también que la unidad de criterios desde la razón como unidad y armonía de voluntades, se comprende como condición indispensable de la convivencia armoniosa, es decir, de la paz. En este contexto la paz, como armonía de voluntades, se pensó en función del acuerdo de las mentes, y el acuerdo de las mentes era posible en función de la existencia de las verdades eternas, manifiestas tanto en la naturaleza como en las Sagradas Escrituras. Y la enseñanza de esas verdades eternas fue la misión de la universidad.

En la época moderna, paz, razón y universidad se piensan de modo distinto. Diferencia que surge del cambio de paradigma frente al mundo, el ser humano y la ciencia propia de esta época, en la cual se propone una nueva base de la razón: la ciencia positivista. Los modernos de este modo rechazaron la identificación de la razón con el orden moral, esto es, con el estudio de las virtudes y las costumbres. A la universidad le corresponde más que nada la producción y difusión del conocimiento científico.

Uno de los mayores representantes y clásicos del pensamiento moderno es Kant, quien intenta elaborar una síntesis de la ciencia con la moral. Con aquella síntesis pudo elaborar un proyecto de paz sin suponer la armonía y la caridad universal. Fue más bien un proyecto de paz de un orden jurídico universal, teniendo como base el respeto de los derechos de todos. La paz no es un ningún estado natural, tiene que ser instituida. Es decir, hay que establecerla como institución, elaborar y respetar su normatividad. Y ello es porque la paz es, es el reino de la ley. Y construir tal realidad es establecer su fuerza moral.

Hay que aclarar, que el pensamiento de Kant puede conducir a quienes pretenden que la universidad aporte a la construcción de la paz hacia una contradicción. Especialmente si desde el rigor académico que brota de esta concepción, se piensa que la universidad y su razón solo puede estudiar la naturaleza y no lo que proviene de la fuerza moral. "Esto pasa en la medida en que la universidad se define como institución dedicada a la razón, y el modelo de la razón deriva de la investigación de la naturaleza, de lo natural. Si además, saber es entender las leyes naturales, y si la construcción de la paz es instituir leyes cuya fuerza sea, en principio, no natural, entonces la universidad no se presta a la construcción de la paz". ${ }^{21}$

Aparece claro, en orden a evitar la anterior contradicción, que se hace necesario superar

\footnotetext{
$\overline{{ }^{20} \text { En este apartado seguimos particularmente a Richards HOWARD }}$, La universidad y la construcción de la paz, en Susy BERMUDEZ (Editora), Estrategias y experiencias para la construcción de la paz. Memorias. Primer encuentro interuniversitario organizado por el Programa de la Universidad de los Andes para la paz - ANPAZ - entre el 24 y el 26 de mayo de 1995, en Santafé de Bogotá, Colombia, Corcas, Bogotá 1996, 177-200.

${ }^{21}$ Ibid179.
} 
el pensamiento moderno, cuya forma clásica es el pensamiento de Kant. Y de hecho es algo que se está superando desde el momento que se niega, desde las nuevas epistemologías, la posibilidad de verdades eternas y de principios eternos y universales sobre los cuales fundar la paz. Se precisa, por lo dicho, una nueva razón, un nuevo acercamiento a la tarea de construir la paz, y una nueva visión de la tarea universitaria. La visión universitaria nueva debe reconocer que el progreso moral siendo necesario, siendo difícil, no depende principalmente de los hallazgos científicos que se pueden realizar en los laboratorios y en las bibliotecas de los campus. Algo si tiene que ver con esos hallazgos. Principalmente, sin embargo, el progreso moral requiere un trabajo de construcción social; no un trabajo de construcción de conocimientos seguido por un trabajo de difusión de los mismos, lo que ha sido el modelo básico de la actividad tradicional universitaria. Y la construcción social, o lo que se debe mejor llama "ciencia constructiva”, define normas por diálogo y por acuerdo.

Lo que exige, al mismo tiempo, un acercamiento mutuo entre la educación popular y la educación universitaria. Porque, de hecho, la educación popular ya funciona con otro modelo de razón, el de la razón dialógica, que es el modelo de racionalidad que ha de caracterizar a la universidad del siglo XXI. Es un modelo que supera el modelo "extensionista", según el cual el especialista sabe y el aprendiz no sabe, modelo cuyo justificativo último es que el especialista representa el saber, saber que él aprendió en la universidad, saber que sigue siendo producto de la razón aplicada a la investigación. Sin embargo, desde el momento que se considera que la paz existe, y que existe más allá de lo jurídico, de lo normativo, sino además en lo cotidiano, y que además tiene que ser construida, pues también es un hecho cultural, se ha de comprender que en la construcción de la paz, la universidad tiene que aprender de la educación popular y de la racionalidad que allí se aplica: la razón dialógica, la razón comunicativa, que en la práctica pedagógica se conoce regularmente como "diálogo de saberes".Pues la verdad, y por lo mismo lo es también la paz, es producto del dialogo, de la comunicación y de la interacción entre las personas.

Pero no solo eso. La universidad ha de aprender también de la educación popular, que la paz no solamente producto de la vida cotidiana, sino que también es experiencia de transformación, de cambio social, de búsqueda de la justicia.

La relación entre paz, razón y universidad, debe llevar a que en la universidad, se respete y valore otras formas de conocimiento científico, tales como el estético, el ético, el religioso, el educativo, el artístico y, fundamentalmente, la revaloración de los conocimientos subyugados u olvidados, como el conocimiento popular. Es un cambio en el modo de concebir el conocimiento humano, con el fin de evitar cualquier fragmentación o cualquier tipo de exclusión de las distintas formas de conocer. Lo que va producir, algo ya dicho acerca de la renovación y modernización de la universidad, la renovación de la relación entre las ciencias naturales y las ciencias sociales, en donde el reto para la universidad de este siglo es ir más allá de la simple coexistencia entre estas dos culturas y lograr una verdadera y auténtica comunicación y diálogo.

Todo lo anterior permite decir que la "razón constructiva”, en el sentido que la paz es tarea constructiva y la ciencia también ha de serlo, es el término que conecta hoy día paz, razón y universidad, desde el "secreto" de esta última. Pues "la paz no es un estado natural del ser humano, y por eso, hay que construirla. La universidad que aporta plenamente a la construcción de la paz se dedicará a la razón en un sentido amplio, no tan sólo a aquella razón que produce el conocimiento objetivo, sino también aquella razón que facilita la 
concertación de los criterios y la acción mancomunada". ${ }^{22}$

Dicha racionalidad abierta, plural, comunicativa y constructora que ha de caracterizar el "secreto de la universidad" hoy día, es igualmente la racionalidad propia de la paz, de lainvestigación para la paz y de la educación para la paz, por lo que paz y universidad se encuentran y se implican mutuamente. De hecho, si se acepta la teoría de Galtung acerca de que los estudios sobre la paz son ciencia social aplicada que comparte con otras ciencias a los seres humanos como seres sociales como objeto de estudio, si bien se centra en el descubrimiento y mecanismos de los conflictos, dicha ciencia como ciencia "trilateral", pues pone en dialogo los hechos, las teorías y los valores, reconoce y hace suyas la pluralidad de racionalidades propias de los paradigmas epistemológicos de hoy. Por lo mismo es una ciencia que trasciende la noción positivista de ciencia, orientándose y favoreciendo más bien "la conjunción de todos los saberes”. Razón por la cual los estudios sobre la paz tienen un carácter interdisciplinario y transdisciplinario. Es decir, han de realizarse a partir de una concepción epistemológica integral, que valora tanto todas las racionalidades, como las formas de conocimiento presentes en todas las culturas, como las formas de conocimientos populares y comunitarios. ${ }^{23}$

\section{Conclusión}

El pensar la relación paz y universidad desde su secreto, es decir la racionalidad, permite abordar los asuntos relacionados con la paz y su educación desde una perspectiva epistemológica y no solamente desde la ética, o peor aún, desde un moralismo o una educación meramente moralizante.
Epistemológicamente se trata, de evitar que la universidad eduque en una racionalidad funcionalista, esencialista y dogmática, tal como señala Alberto Parra en sus indagaciones sobre la paz real. Contraria, según el, a la racionalidad hermenéutica abierta al examen y a la interpretación. ${ }^{24}$

La primera es una racionalidad que alimenta la fragmentación del ser y del saber, el encerramiento en carreras y profesiones, el saber monológico y el supeditar el saber a las lógicas del mercado. En el ámbito de las personas, continua Parra, crea subjetividades e individualidades cerradas, solas, egoístas, narcisistas, incomunicadas, fragmentadas. En el ámbito social yuxtaposición de personas, la masa, el anonimato, la soledad, el impersonalismo y la inexistencia de horizontes comunes. En el modo de pensar y de conocer sus consecuencias son la negación del pensamiento libre, la subyugación del estudiante al docente, el mandamiento irracional del orden establecido, el despotismo.

Este tipo de racionalidad es una racionalidad neutra. En palabras de Parra, significa una racionalidad poco sensible, alejada de los intereses de la vida, academicista, distractora de la conciencia cívica y política.

Por el contrario, la racionalidad hermenéutica es interrelacional e intersubjetiva, creadora y potenciadora de visiones de conjunto, favorece la convergencia y la interacción de saberes, el acercamiento al otro, el establecimiento de comunidades de dialogo y de comunicación. Este tipo racionalidad esta a la base del conocimiento para la convivencia y la justicia social.

Por todo ello, concluye Parra, y apoyados en palabra concluye este estudio, "la verdadera paz social se gesta en la academia".

\footnotetext{
${ }^{23}$ Johan GALTUNG, Fundamentos de estudios sobre la paz", en Ana RUBIO, Presupuestos teóricos y éticos sobre la paz. Seminario de la paz y los conflictos. Granada, Universidad de Granada 1993, 19.

${ }^{24}$ Alberto PARRA, Violencia total y paz real. Indagaciones teológicas, Pontificia Universidad Javeriana, Bogotá 2010.
} 


\section{Referencias bibliográficas}

Alberto PARRA, Violencia total y paz real. Indagaciones teológicas, Pontificia Universidad Javeriana, Bogotá 2010.

Alfonso FERNÁNDEZ HERRERÍA, La educación para la paz en la universidad, en Javier Rodríguez Alcázar (editor), Cultivar la paz. Perspectivas desde la universidad de Granada, Universidad de Granada, Granada 2000.

Ángelo PAPACCHINI, Universidad, guerra y paz, en Carmen Lucia DIAZ Claudia MOSQUERA - Fabio FAJARDO (Compiladores), La universidad piensa la paz: obstáculos y posibilidades, Universidad Nacional de Colombia, Bogotá 2002, 15-49.

Antanas MOCKUS, Lugar de la pedagogía en la universidad, en Universidad Nacional de Colombia, Reforma académica. Documentos, Universidad Nacional de Colombia, Bogotá 1995, 9-24.

Antanas MOCKUS SIVICKAS, Fundamentos teóricos para una reforma de la universidad, en ESCUELA SUPERIOR DE ADMINISTRACIÓN PUBLICA, Reflexiones sobre el sentido de la universidad, ESAP, Bogotá 1992, 97 - 125.

\section{ASOCIACIÓN COLOMBIANA DE UNI-}

46 VERSIDADES (ASCUN), La universidad y la paz. Consejo nacional de rectores 30 de septiembre - 1 de octubre, 1999, ASCUN, Santafé de Bogotá 1999.

ASOCIACIÓN COLOMBIANA DE UNIVERSIDADES POR LA PAZ (ASCUN) - RED DE UNIVERSIDADES POR LA PAZ (REDEUNIPAZ), Primer congreso universitario por la paz, tomo I y II, Santa fe de Bogotá D.C. 12, 13 y b14 de mayo de 1999, ASCUN, Santa fe de Bogotá D.C., 2000.
Cristina DE LA CRUZ AYUSO - Perú SASIA SANTOS, La responsabilidad de la universidad en el proyecto de construcción de una sociedad, en "Revista educación superior y sociedad", Número 2, septiembre de 2008, $17-52$.

Francisco NAISHTAT - Francisco GARCIA RAGGIO - Ana María GARCIA RAGGIO - Susana VILLAVICENCIO (compiladores), Filosofías de la universidad y conflicto de racionalidades, Ediciones Colihue, Buenos Aires 2001.

François VALLAEYS, Responsabilidad social universitaria: una nueva filosofía de gestión ética e inteligente para las universidades, en "Revista educación superior y sociedad", Número 2, septiembre de 2008, 191-220.

Guillermo HOYOS VASQUEZ, La universidad como comunidad y como institución, en ESCUELA SUPERIOR DE ADMINISTRACIÓN PUBLICA, Reflexiones sobre el sentido de la universidad, ESAP, Bogotá 1992, 17 - 42.

Guillermo HOYOS, El ethos de la universidad, en "Revista UIS-Humanidades 1 (1998) $13-23$.

Guy-Réal THIVIERGE, Au coer de l'universitácatholique: Leducation a la paix, en "Seminarium" 3/4 (2001) 797 - 824.

Jacques MARCOVICH, La universidad (im) posible, Cambridge UniversityPress Organización de Estados Americanos (OEI), Madrid 2002.

Johan GALTUNG, Fundamentos de estudios sobre la paz", en Ana RUBIO, Presupuestos teóricos y éticos sobre la paz. Seminario de la paz y los conflictos. Granada, Universidad de Granada 1993.

Lucia DIAZ CARMEN - Claudia MOSQUERA - Fabio FAJARDO (Compiladores), La universidad piensa la paz: obstáculos 
y posibilidades, Universidad Nacional de

Colombia, Bogotá D.C. 2002.

Mariana DELGADO BARÓN - Yaneth Angélica VARGAS PEDRAZA - Ivonne RAMOS HENDEZ, Los retos de la responsabilidad social universitaria. Construyendo paz desde la universidad, en Revista educación superior y sociedad", Número 2, septiembre de 2008, 64 -91.

Maria Laura FERRARI, Pensare quello che facciamo. "Esercitare" ilpensiero per formare alla non-violenza, en "Encyclopaideia" 11 (2002) 65-85.

Paulo FREIRE, Política y educación, Siglo Veintiuno editores, Buenos Aires 1996, 122 132.

Ricardo LUCIO A., la pedagogía y la didáctica en el quehacer universitario, en ESCUELA SUPERIOR DE ADMINISTRACIÓN PUBLICA, Reflexiones sobre el sentido de la universidad, ESAP, Bogotá 1992, 77- 94.

Richards HOWARD, La universidad y la construcción de la paz, en Susy BERMUDEZ (Editora), Estrategias y experiencias para la construcción de la paz. Memorias. Primer encuentro interuniversitario organizado por el Programa de la Universidad de los Andes para la paz - ANPAZ - entre el 24 y el 26 de mayo de 1995, en Santafé de Bogotá, Colombia, Corcas, Bogotá 1996, 177-200.

Xesus JARES, Educación para la paz y organización escolar, en Alfonso FERNÁNDEZ HERRERÍA (editor), Educación para la paz: nuevas propuestas, Universidad de Granada, Granada 1994, 285 $-316$ 